\title{
A YOUNG-LIKE INEQUALITY WITH APPLICATIONS TO THE COMMUTATOR ESTIMATES
}

\author{
JIANG XU
}

\begin{abstract}
In the recent decade, Fourier analysis techniques based on the Littlewood-Paley decomposition have proved to be very efficient in the study of PDEs, since J.-M. Bony introduced the paradifferential calculus. Of those techniques, commutator estimates play the crucial role in dealing with bilinear estimates. In this paper, we develop a Young-like inequality which is the generalization of the classical Young's convolution inequality. The new inequality enables us to obtain various commutator estimates in the $L^{p}$-framework, which creates the basis to obtain different nonlinear a priori estimates in the analysis of PDEs.
\end{abstract}

Mathematics subject classification (2010): 35Q30, 76D05, 35Q35, 42B35.

Keywords and phrases: Young's inequality, Littlewood-Paley decomposition, commutator estimates, Bony's paradifferential calculus.

\section{REFERENCES}

[1] H. ABIDI, Equation de Navier-Stokes avec densité et viscosité variables dans l'espace critique, Revista Math. Iber., 23, 2 (2007), 537-586.

[2] H. ABIDI, G. L. GUi AND P. ZhANG, On the wellposedness of three-dimensional inhomogeneous Navier-Stokes equations in the critical spaces, Arch. Ration. Mech. Anal., 204, 1 (2012), 189-230.

[3] H. Bahouri, J. Y. Chemin And R. Danchin, Fourier Analysis and Nonlinear Partial Differential Equations, Grundlehren der mathematischen Wissenschaften, Berlin: Springer-Verlag, 2011.

[4] J.-Y. CHEMIN AND N. LERNER, Flot de champs de vecteurs non lipschitziens et équations de NavierStokes, J. Differential Equations, 121, 2 (1995), 314-328.

[5] Q. L. Chen, C. X. MiAO And Z. F. Zhang, Global well-posedness for the compressible NavierStokes equations with the highly oscillating initial velocity, Comm. Pure Appl. Math., 63, 9 (2010), 1173-1224.

[6] Q. L. Chen, C. X. MiaO AND Z. F. Zhang, Well-posedness in critical spaces for the compressible Navier-Stokes equations with density dependent viscosities, Rev. Mat. Iberoamericana, 26, 3 (2010), 915-946.

[7] R. DANCHIN, Global existence in critical spaces for compressible Navier-Stokes equations, Inventiones Mathematicae, 141, 3 (2000), 579-614.

[8] R. Danchin, Global existence in critical spaces for flows of compressible viscous and heatconductive gases, Arch. Ration. Mech. Anal., 160, 1 (2001), 1-39.

[9] R. DANCHIN, On the well-posedness of the incompressible density-dependent Euler equations in the $L^{p}$ framework, J. Differential Equations, 248, 8 (2010), 2130-2170.

[10] R. DANChIN AND F. FANELLI, The well-posedness issue for the density-dependent Euler equations in endpoint Besov spaces, J. Math. Pures Appl., 96, 3 (2011), 253-278.

[11] L. Grafakos, Classical Fourier Analysis (2nd), New York: Springer, 2008.

[12] C. C. HAO AND H. L. LI, Well-posedness for a multidimensional viscous liquid-gas two phase flow model, SIAM J. Math. Anal., 44, 3 (2012), 1304-1332.

[13] M. Paicu And P. Zhang, Global solutions to the 3-D incompressible anisotropic Navier-Stokes system in the critical spaces, Commun. Math. Phys., 307, 3 (2011), 713-759.

[14] M. PAICU AND P. ZHANG, Global solutions to the 3-D incompressible inhomogeneous Navier-Stokes system, J. Funct. Anal., 262, 8 (2012), 3556-3584. 
[15] J. Z. QIAN AND Z. F. ZHANG, Global well-posedness for compressible viscoelastic fluids near equilibrium, Arch. Ration. Mech. Anal., 198, 3 (2010), 835-868.

[16] J. XU, Relaxation-time limit in the isothermal hydrodynamic model for semiconductors, SIAM J. Math. Anal., 40, 5 (2008), 1979-1991.

[17] J. XU, Global classical solutions to the compressible Euler-Maxwell equations, SIAM J. Math. Anal., 43, 6 (2011), 2688-2718.

[18] J. XU AND Z. J. WANG, Relaxation limit in Besov spaces for compressible Euler equations, J. Math. Pures Appl., 99, 1 (2013), 43-61.

[19] T. ZHANG, Global wellposed problem for the 3-D incompressible anisotropic Navier-Stokes equations in an anisotropic space, Commun. Math. Phys., 287, 1 (2009), 211-224.

[20] T. Zhang AND D. Y. FANG, Global existence of strong solution for equations related to the incompressible viscoelastic fluids in the critical $L^{p}$ framework, SIAM J. Math. Anal., 44, 4 (2012), 2266-2288.

[21] T. ZhANG AND D. Y. FANG, Global wellposed problem for the 3-D incompressible anisotropic Navier-Stokes equations, J. Math. Pures Appl., 90, 5 (2008), 413-449. 